\title{
Empty-nest elderly: mahjong and Chinese square dance for improving the cognitive function in the community
}

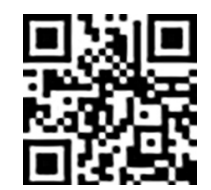

Original article

Yue Zhanga ${ }^{a}$ Marcia A. Petrinia,b,*

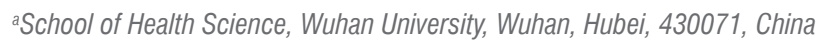

${ }^{b}$ Chiang Mai University, Chiang Mai, Thailand

Received: 30 January 2018; Accepted: 23 March 2018; Published: 20 March 2019

Abstract: Objective: The elderly population has proliferated worldwide. The empty-nest family pattern has become predominant among the aging people, and they are more vulnerable to the development of cognitive disorders. However, there is no standardized service in the community nursing care that includes procedures on how to improve the cognitive function of the elderly. Meanwhile, the booming number of empty-nest elderly stimulates the community nurses to assume the responsibility for their care. All of these bring more difficulties and opportunities for community nurses who are dedicated to the prevention of geriatric cognitive disorders.

Methods: The authors reviewed the literature related to "empty-nest elderly", "cognitive function", "mahjong", and "Chinese square dance" in the Elsevier, Web of Science (WOS), China National Knowledge Infrastructure (CNKI), Springer and PubMed databases. The study illustrates the utility possibility of an efficient and straightforward method for improving the cognitive function among the elderly in the context of community nursing care in China and even in the rest of the world.

Results: Mental and physical activity contributes to cognitive fitness and may be beneficial in delaying cognitive decline. Mental activities, such as playing mahjong, and physical activities, such as the Chinese square dance, are common Chinese activities. Both of them can affect cognitive function in some way.

Conclusions: China is experiencing one of its most severe aging problems. Community health personnel and related professionals may consider using mahjong and Chinese square dance to promote psychological health in empty-nest elderly individuals in the community.

Keywords: empty-nest elderly • cognitive function • mahjong • Chinese square dance

(c) Shanxi Medical Periodical Press.

\section{Introduction}

In 2016, the United States Census Bureau reported that the elderly population will rise by 55 million in the ensuing 2 years and account for $8.5 \%$ of the total population. The number of people aged 65 years and older will increase by about 236 million in the next 9 years, and it will almost double to 1.6 billion globally from 2025 to 2050 , while the total population will increase just $34 \%{ }^{1}$

The elderly population has grown rapidly worldwide, and the aging problem has become serious. China is experiencing one of its most severe aging problems. The United States Census Bureau represented the elderly

Citation information: Zhang Y, Petrini MA. Empty-nest elderly: mahjong and Chinese square dance for improving the cognitive function in the community. Front Nurs. 2019; 1: xx-xx.

○ Open Access. ๑ 2019 Yue Zhang and Marcia A. Petrini, published by Sciendo. (c) Br-Nc-ND This work is licensed under the Creative Commons Attribution NonCommercial-NoDerivatives 4.0 License. 
population in 2015 as $10.1 \%$ of the total population; this figure will increase to $17.2 \%$ in 2030 and $26.7 \%$ in 2050 in China. By 2050, the elderly population in China (348.8 million) will be more than the combined total populations of Japan, Egypt, Germany, and Australia (345.6 million). ${ }^{1}$

Gratton and Gutmann ${ }^{2}$ indicated that the percentage of empty-nest married men aged 60 years and older living with their wife increased from $19 \%$ to $78 \%$ between 1880 and 2000 in the United States. Empty nest families have been becoming the main pattern among aging people. ${ }^{3}$ $\mathrm{Li}$ et al. ${ }^{4}$ reported that the number of empty-nest elderly families will reach $90 \%$ of the total number of families in China. Many studies exploring empty nesters' cognitive condition in China have shown that this kind of population is more vulnerable to depression, loneliness, dementia, Alzheimer's disease, and other mental syndromes. Some of the studies indicated that the rural area is more vulnerable. Some studies have reported that psychological and physical activity contributes to cognitive fitness and may be beneficial in delaying cognitive decline.

In China, mental activities such as mahjong and physical activities such as Chinese square dance are the most common activities. However, there has been little previous research into mahjong and Chinese square dance. Researchers $^{5-7}$ have reported that mahjong can affect the cognitive function of people with dementia and can preserve the functioning or delay the decline in certain cognitive domains, even in those people with significant cognitive impairment. Researchers ${ }^{8,9}$ showed that Chinese square dance can promote cognitive function in middleaged females, in addition to relieving depression and anxiety in old adults. Many studies indicated that dancing could improve physical fitness, mental health, and creativity; help develop social skills and provide entertainment well during aging; release emotional expression; and decrease stress, depression, loneliness, and dementia. Cultural dance can increase the physical activity and health. ${ }^{10}$

Therefore, investigating whether mahjong and Chinese square dance improve the cognitive function in community empty-nest elderly or not is appropriate. The study is significant in the context of understanding the needs of elderly adults in ways that can contribute to a public policy aimed at improving the cognitive function of the empty nester, enabling China to handle the challenges rapidly and cost-effectively in population aging.

\section{Cognitive condition of the empty-nest elderly}

\subsection{Cognitive condition of the empty-nest elderly worldwide}

A couple of studies ${ }^{11,12}$ have reported that empty nesters experience loneliness after their children leave, but the participants in both studies were midlife women. Jakobsson and Hallberg ${ }^{13}$ indicated that loneliness and fear are universal among aging people. Fahrenberg ${ }^{12}$ termed the depression and isolation found among empty nesters the "empty nest syndrome".

Depression is one of the most frequent mental disorders among aging people, and it severely impairs their lives. The risk factors include female sex, widowhood or single life, low socioeconomic status, bereavement, stressful life events, poor social support, prior depression, comorbidities, disability, cognitive deterioration, and vascular factors. ${ }^{14,15}$ Depression comes ninth behind prolific killers when ranked by disability and death combined in the world. ${ }^{16}$

Reitz and Mayeux ${ }^{17}$ showed that the global prevalence of dementia is as high as 24 million, and it has been predicted to quadruple by the year 2050. Aging is one of the risk factors for dementia and Alzheimer's disease. The incidence and prevalence increase with age, almost doubling every 5 years after the age of 60 years. ${ }^{18}$ The initial no-symptoms preclinical phase of Alzheimer's disease is followed by a period wherein cognitive impairment, but no functional impairment, is present (mild cognitive impairment). ${ }^{19}$ Among aging Malays, the prevalence of any cognitive impairment has been reported to be $25.5 \% .^{20}$

\subsection{Cognitive condition of the empty-nest elderly in China}

Chinese empty nesters are vulnerable to many common cognitive problems as are empty nesters worldwide. Gao et al. ${ }^{21}$ showed that empty nesters' ability of self-care was worse than and the physical and mental scores were lower than the non-empty nester. Lyu et al. ${ }^{22}$ showed that the empty nester was statistically low in vitality, emotional role, and mental health. Liu and $G^{23}{ }^{23}$ indicated that the empty nester was vulnerable to mental problems and was prone to have low level of life satisfaction.

Feng et al. ${ }^{24}$ noted that the high prevalence of geriatric depression among Uyghur elderly in Xinjiang is serious: $10.61 \%$ among the elderly experienced depression. Female sex, low income, low education level, chronic diseases, experienced adverse events during the past 2 years, and the lack of ability to take self-care are the risk factors. ${ }^{24} \mathrm{Ma}$ et al.$^{25}$ indicated that empty nesters show a higher prevalence of depressive symptoms. Su et al. ${ }^{26}$ showed that the empty nester in the rural area has more depression and less objective support than an urban empty nester. Tong et al. ${ }^{27}$ indicated that in Shanghai, $>30 \%$ of the elderly reported a mild or above-mild level of depression. Participants with low income, less favorable housing conditions, and 
loneliness showed more depressive symptoms. Wang et al. ${ }^{28}$ reported that elderly adults with depression, loneliness, and cognitive impairment, as well as females and people living alone or in rural areas, were at risk for anxiety disorder. The prevalence of depressive symptoms was $74.46 \%$ among empty nesters in the rural area of Yongzhou City, and the depressive symptoms showed association with negative coping style, support utilization, religious beliefs, economic status, marital status, and subjective support. ${ }^{29}$ Zhai et al. ${ }^{3}$ revealed that it is natural for empty nesters who are male, $\geq 70$ years old, and retired, as well as those with low level of education, to exhibit depression. Zhang et al. ${ }^{30}$ reported that in the economically depressed area of Hunan Province, elderly persons' education, income, and social support showed significantly more effects in predicting subjective well-being.

Cheng et al. ${ }^{31}$ indicated that loneliness is obvious in the empty nester and that depressive symptoms constitute the stronger risk factor. Liu and $\mathrm{Guo}^{32}$ reported that the empty nester has a higher level of loneliness. Social support and income are negatively associated, whereas the education level and being single are positively associated. Wu et al. ${ }^{33}$ revealed that $80.94 \%$ of the empty nesters in Anhui rural area had moderate-to-high levels of loneliness.

Chan et al. ${ }^{34}$ indicated that the number of people with dementia and Alzheimer's disease has been increasing fast in the past few years in China. The number of elderly with dementia was 3.68 million in 1990, 5.62 million in 2000, and 9.19 million in 2010. And the number for those with Alzheimer's disease was 1.93 million in 1990, 3.71 million in 2000, and 5.69 million in 2010 . Dong et al. ${ }^{35}$ reported that in the period 1980-2004, Alzheimer's disease and vascular dementia were the major forms of dementia in China. Moreover, the risk factors for Alzheimer's disease are gender, education level, occupation, and age. Hua and $\mathrm{Jin}^{36}$ indicated that the quality of life of the immediate family members of senile dementia patients is relatively low. China's empty nesters need better care, especially those in rural areas. ${ }^{37}$

\section{Benefits of mental and physical activities in the aging population}

\subsection{Mental activities}

The video game experts possess higher speeds and better visual short-term memory, switch more quickly, and mentally rotate objects more efficiently. ${ }^{38}$ Hardy et al. ${ }^{39}$ indicated that the participants who were trained through mental activities had more significant improvement in terms of the speed of processing, short-term memory, working memory, problem solving, fluid reasoning assessments, and cognitive function. Spiritual activity may benefit the elderly adults' cognitive function. ${ }^{40}$ Digital games are used to enhance the cognitive function. ${ }^{41}$ Mental training can strengthen plasticity in the brain and psychological function, in addition to increasing control over the distribution of limited brain resources. ${ }^{42}$

\subsection{Physical activities}

Fox ${ }^{43}$ revealed that exercise is effective in depression, has a positive influence on physical self-perceptions and self-esteem, and has a moderate anxiety-reducing effect. Health promotion behaviors change the quality of life and overall functioning among Korean communitydwelling aging people, and the inclusion of exercise in health promotion behaviors is essential. ${ }^{44} \mathrm{~A}$ meta-analysis indicated that fitness has momentous but selective benefits on cognitive function. ${ }^{19}$

Dance - as a form of physical activity - helps release emotional expression, allows for creativity, and decreases stress, depression, and loneliness. Moreover, it helps the older brain to form new interconnections and to work faster, in addition to increasing the improvement of memory, attention, and the ability to multitask and plan. ${ }^{45}$ Dancing can improve physical fitness and mental health, develop social skills, and provide entertainment during aging. ${ }^{46}$ Dance can prevent Alzheimer's disease and other forms of dementia in the elderly. Participants who engaged in reading, dancing, and playing board games and a musical instrument at least 11 times per month reduced their risk of dementia by $63 \% .{ }^{47}$

\subsection{Mental and physical activities}

Klusmann et al. ${ }^{48}$ reported that exercise and computer class have equivalent beneficial effects on cognitive fitness and might delay cognitive decline in healthy elderly women. Exercise enhances cognitive vitality across the life span through reduction of disease risk and improvement in the molecular and cellular structure and function of the brain. ${ }^{49}$ Mental exercise is hypothesized to offer personal benefits to the cognitive function, the benefits becoming higher with increased age. ${ }^{50}$

\section{Reasons for applying mahjong and Chinese square dance}

\subsection{Reasons for applying mahjong as a mental activity}

Mahjong is a psychological game that originated in China during the period of the Qing dynasty, and it involves four people. It is very popular in China, even worldwide. The main population playing mahjong in China is made up of 
the old adults. Mahjong is easy to learn but is also a profoundly mentally demanding game. Mahjong requires the player to calculate the composite scores, remember others' moves, and build up one's own strategy during the play. Mahjong does not require professional supervision and can be implemented as widely as space allows at a given time.

Mahjong can affect the cognitive function of people with dementia, even after withdrawal for a month. Once an initial threshold is attained, the benefits of mahjong appear to be long-lasting. It also can be a potentially benefit for communities in terms of expenditure when mahjong is used in daily routine care. ${ }^{5}$ Mahjong can preserve functioning or delay decline in specific cognitive domains, even in those with significant cognitive impairment. ${ }^{6}$ One study showed that playing mahjong can enhance shortterm memory, attention, and logical reasoning capability in the middle-aged and elderly populations. Playing mahjong yields superior benefits over time. ${ }^{7}$

\subsection{Reasons for applying Chinese square dance as a physical activity}

Chinese square dance is an exercise routine performed to music in squares, parks, and other public places. Dancing originating in China has been recorded during the reign of Emperor Yao. There are $>8000$ kinds of Chinese square dances, and they come in a variety of levels of difficulty. It is very popular with middle-aged and

\section{References}

1. United States Census Bureau. An Aging World: 2015. Washington: US Government Publishing Office report; 2016.

2. Gratton B, Gutmann MP. Emptying the nest: older men in the United States, 1880-2000. Popul Develop Rev. 2010;36:331-356.

3. Zhai Y, Yi H, Shen W, et al. Association of empty nest with depressive symptom in a Chinese elderly population: a cross-sectional study. J Affect Disorders. 2015;187:218-223.

4. Li DM, Chen TY, Li GY. The problem of mental health in the elderly in empty-nest family. Chin $J$ Gerontol. 2003;23:405-407 (in Chinese).

5. Cheng ST, Chan AC, Yu EC. An exploratory study of the effect of mahjong on the cognitive functioning of persons with dementia. Int Geriatr Psych. 2006;21:611-617.

6. Cheng ST, Chow PK, Song YQ, et al. Mental and physical activities delay cognitive decline in older persons with dementia. Am J Geriatr Psychiatry. 2014;22:63-74. old-aged women. On August 6, 2016, 1000 women gathered to dance at the Xian Yang Unity Square in Shaanxi Province, Xianyang City, for the World Fitness day.

One related article showed that Chinese square dance could effectively reduce the depression levels of women in the perimenopausal stage. ${ }^{8}$ Chinese square dance can promote cognitive function and relieve depression and anxiety in old adults. ${ }^{9}$

\section{Conclusions}

The aging problem has become increasingly serious. Empty-nest elderly is the main pattern of old adults. Mental and physical activity can change their mental syndrome (depression, loneliness, dementia, Alzheimer's disease, and so on). Mahjong and Chinese square dance seem to open a method for community nurse care. Further research is needed to develop these tools to cater to the needs of empty-nest elderly and community nurses. Thus, the cognitive function of the elderly population will be more easily promoted. Community health personnel and related professionals may consider mahjong and Chinese square dance to promote psychological health in empty-nest elderly individuals in a community.

\section{Conflicts of interest}

All contributing authors declare no conflicts of interest.

7. Chu-Man L, Chang M, Chu M. Effects of mahjong on the cognitive function of middle-aged and older people. Int J Geriatr Psych. 2015;30:995-997.

8. Gao L, Zhang L, Qi H, Petridis L. Middle-aged female depression in perimenopausal period and square dance intervention. Psychiatr Danub. 2016;28:372-378.

9. Zhang XA, Ni XM, Liu F. Study about effects of square dancing on the cognitive function and emotion of the aged. Chin Foreign Med Res. 2012;10:4-5.

10. Sheila J, David RB. Cultural dance: an opportunity to encourage physical activity and health in communities. Am J Health Educ. 2001;32:4, 216-222.

11. Dennerstein L, Dudley E, Guthrie J. Empty nest or revolving door? A prospective study of women's quality of life in midlife during the phase of children leaving and re-entering the home. Psychol Med. 2002;32:545-550.

12. Fahrenberg B. Coping with the empty nest situation as a developmental task for the aging female-an 
analysis of the literature. Z Gerontol. 1986;19:323335 (in German).

13. Jakobsson U, Hallberg IR. Loneliness, fear, and quality of life among elderly in Sweden: a gender perspective. Aging Clin Exp Res. 2005;17:494-501.

14. Helmer C, Montagnier $D$, Pérès K. Descriptive epidemiology and risk factors of depression in the elderly. Psychol Neuropsychiatr Vieil. 2004;2 Suppl 1:S7-S12.

15. Lépine JP, Bouchez S. Epidemiology of depression in the elderly. Int Clin Psychopharmacol. 1998;13 Suppl 5:S7-S12.

16. Smith K. Mental health: a world of depression. Nature. 2014; 515:181.

17. Reitzn C, Mayeux R. Alzheimer disease: epidemiology, diagnostic criteria, risk factors and biomarkers. Biochem Pharmacol. 2014;88:640-651.

18. Santana I, Farinha F, Freitas S, Rodrigues V, Carvalho $\AA$. The epidemiology of dementia and Alzheimer disease in Portugal: estimations of prevalence and treatment-costs. Acta Med Port. 2015;28:182-188 (in Portuguese).

19. Colcombe S, Kramer AF. Fitness effects on the cognitive function of older adults: a meta-analytic study. Psychol Sci. 2003;14:125-130.

20. Hilal S, Tan CS, Xin S, Amin SM, et al. Prevalence of cognitive impairment and dementia in Malays epidemiology of dementia in Singapore study. Curr Alzheimer Res. 2015;14:620-627.

21. Gao L, Cao X, Zhang M. The study on community health education of empty nest elderly. Engineering. 2013;05:137-139.

22. Lyu XL, Jiang $Y H$, Sun $Y H$, et al. Short form 36-Item Health Survey test result on the empty nest elderly in China: a meta-analysis. Arch Gerontol Geriat. 2013;56:291-297.

23. Liu LJ, Guo Q. Life satisfaction in a sample of empty-nest elderly: a survey in the rural area of a mountainous county in China. Qual Life Res. 2008;17:823-830.

24. Feng L, Li P, Lu C, et al. Burden and correlates of geriatric depression in the Uyghur elderly population, observation from Xinjiang, China. PLoS One. 2014;9:114-139.

25. Ma Y, Fu H, Wang JJ, et al. Study on the prevalence and risk factors of depressive symptoms among 'empty-nest' and 'non-empty-nest' elderly in four provinces and cities in China. Chin J Epidemiol. 2012;33:478-482 (in Chinese).

26. Su D, Wu XN, Zhang $Y X$, et al. Depression and social support between China' rural and urban empty-nest elderly. Arch Gerontol Geriat. 2012;55:564-569.
27. Tong HM, Lai DW, Zeng Q, Xu WY. Effects of social exclusion on depressive symptoms: elderly Chinese living alone in Shanghai, China. J Cross Cult Gerontol. 2011;26:349-364.

28. Wang Z, Shu D, Dong B, Luo L, Hao Q. Anxiety disorders and its risk factors among the Sichuan empty-nest older adults: a cross-sectional study. Arch Gerontol Geriat. 2013;56:298-302.

29. Xie LQ, Zhang JP, Peng F, Jiao NN. Prevalence and related influencing factors of depressive symptoms for empty-nest elderly living in the rural area of YongZhou, China. Arch Gerontol Geriat. 2010;50:24-29.

30. Zhang JP, Huang HS, Ye M, Zeng H. Factors influencing the subjective well being (SWB) in a sample of older adults in an economically depressed area of China. Arch Gerontol Geriat. 2008;46:335-347.

31. Cheng $P$, Jin $Y$, Sun $H$, et al. Disparities in prevalence and risk indicators of loneliness between rural empty nest and non-empty nest older adults in Chizhou, China. Geriat Gerontol Int. 2015:15:356-364.

32. Liu LJ, Guo Q. Loneliness and health-related quality of life for the empty nest elderly in the rural area of a mountainous county in China. Qual Life Res. 2007;16:1275-1280.

33. Wu ZQ, Sun L, Sun YH, Zhang XJ, Tao FB, Cui GH. Correlation between loneliness and social relationship among empty nest elderly in Anhui rural area, China. Aging Ment Health. 2010;14:108-112.

34. Chan KY, Wang W, Wu JJ, et al. Epidemiology of Alzheimer's disease and other forms of dementia in China, 1990-2010: a systematic review and analysis. Lancet. 2013; 381: 2016-2023.

35. Dong MJ, Peng B, Lin XT, Zhao J, Zhou YR, Wang $\mathrm{RH}$. The prevalence of dementia in the People's Republic of China: a systematic analysis of 19802004 studies. Age Ageing. 2007;36:619-624.

36. Hua YU, Jin Y. Qol of immediate family members of senile dementia patients and its influencing factors. Chin General Pract. 2013;16:171-175 (in Chinese).

37. Gaon YL, Wei YB, Shen YD, Tang YY, Yang JR. China's empty nest elderly need better care. J Am Geriatr Soc. 2014;62:1821-1822.

38. Boot WR, Kramer AF, Simons DJ, Fabiani M, Gratton $G$. The effects of video game playing on attention, memory, and executive control. Acta Psychol (Amst). 2008;129:387-398.

39. Hardy JL, Nelson RA, Thomason ME, et al. Enhancing cognitive abilities with comprehensive training: a large, online, randomized, active-controlled trial. PLoS One. 2015;10:e134467.

40. Fung AW, Lam LC. Spiritual activity is associated with better cognitive function in old age. East Asian Arch Psychiatry. 2013;23:102-108. 
41. Schott G, Hodgetts D. Health and digital gaming: the benefits of a community of practice. $J$ Health Psychol. 2006;11:309-316.

42. Slagter HA, Lutz A, Greischar LL, et al. Mental training affects distribution of limited brain resources. PLoS Biol. 2007;5:e138.

43. Fox KR. The influence of physical activity on mental well-being. Public Health Nutr. 1999;2:411-418.

44. Lee Y. The predictive value of self assessed general, physical, and mental health on functional decline and mortality in older adults. J Epidemiol Community Health. 2000;54:123-129.

45. Alpert PT. The health benefits of dance. Home Health Care Manag Pract. 2011; 23:155-157.

46. Bremer Z. Dance as a form of exercise. Br J General Pract. 2007;57:166.
47. Verghese J, Lipton RB, Katz MJ, et al. Leisure activities and the risk of dementia in the elderly. $N$ Engl $J$ Med. 2003;348:2508-2516.

48. Klusmann V, Evers A, Schwarzer R, et al. Complex mental and physical activity in older women and cognitive performance: a 6-month randomized controlled trial. J Gerontol A Biol Sci Med Sci. 2010;65:680-688.

49. Kramer AF, Erickson KI. Capitalizing on cortical plasticity: influence of physical activity on cognition and brain function. Trends Cogn Sci. 2007;11:342-348.

50. Salthouse TA. Mental exercise and mental aging: evaluating the validity of the "use it or lose it" hypothesis. Perspect Psychol Sci. 2006;1:68-87. 